\title{
Correlation of Temporal Parameters of Laryngeal Excursion by Using Force-Sensing Resistor Sensors with Hyoid Motion in Videofluoroscopic Swallowing Study
}

\author{
Chin-Man Wang ${ }^{1} \cdot$ Chao-Jan Wang $^{2} \cdot$ Wann-Yun Shieh $^{3} \cdot$ Yen-Chia Chen ${ }^{1} \cdot$ Wei-Jen Cheng ${ }^{1} \cdot$ Wei-Han Chang $^{1}$
}

Received: 28 January 2020 / Accepted: 23 April 2020 / Published online: 28 April 2020

(c) The Author(s) 2020

\begin{abstract}
Small flexible force-sensing resistor (FSR) sensors can detect laryngeal excursion during swallowing, but the detected laryngeal excursion has not been correlated with videofluoroscopic swallowing study (VFSS) results. Here, we tested the correlation of temporal parameters between the laryngeal excursion recording by FSR sensor and the hyoid motion recording by VFSS under simultaneously swallowing test recordings. Swallowing measurements were recorded in a radiological suite by simultaneously using VFSS and FSR sensors to detect hyoid motion and laryngeal excursion, respectively. Volunteers sat with their head vertical to the Frankfort plane. Two FSR sensors, each for detecting thyroid cartilage excursion and thumb pressing, were placed. VFSS images and FSR sensor signals during single 5-mL barium liquid (30\% wt/volume \%) bolus swallowing were collected and analyzed for four swallows per participant. In total, 15 men (28.0 \pm 4.1 years old); 14 women $(28.4 \pm 4.2$ years old) were recruited. Temporal parameters between VFSS and noninvasive system demonstrated a strong correlation by Pearson's correlation analysis: in men $(R=0.953-0.999)$ and in women $(R=0.813-0.982)$, except for VT1-V1 compared with FT1-F1, which demonstrated a moderate correlation in women $(R=0.648$; all $p<0.001)$. Only VT1-V1 and FT1-F1 in women displayed a significant difference $(p=0.001)$. Therefore, this is the first study to simultaneous record VFSS and noninvasive signals by FSR sensor. The correlation of temporal parameters between these two tests was strong. This finding is valuable for future applications of this noninvasive swallowing study tool.
\end{abstract}

Keywords Deglutition · Deglutition disorders $\cdot$ Swallowing temporal parameters $\cdot$ FSR $\cdot$ VFSS $\cdot$ Correlation

\section{Introduction}

Noninvasive swallowing studies, a new trend, have several advantages. Because it does not involve radiation exposure, it is suitable for studying early, subtle, or mild dysphagia $[1,2]$, and is favorable for collecting large amount of

Chin-Man Wang

cmw1314@cgmh.org.tw

Wann-Yun Shieh

wyshieh@mail.cgu.edu.tw

Chao-Jan Wang

cjwang@cgmh.org.tw

Yen-Chia Chen

mr2422@cgmh.org.tw

Wei-Jen Cheng

weijenpaul@gmail.com

Wei-Han Chang

mr7252@cloud.cgmh.org.tw swallowing data, particularly if short term follow-ups and repeated measurements are necessary [3]. Furthermore, noninvasive swallowing measurements could be useful for differentiating normal and pathological swallowing physiology in the future.

1 Department of Physical Medicine and Rehabilitation, Linkou Chang Gung Memorial Hospital, College of Medicine, Chang Gung University, Gueishan District, No.5, Fu-Hsing Street, Taoyuan City 33305, Taiwan R.O.C.

2 Department of Medical Image and Intervention, Linkou Chang Gung Memorial Hospital, College of Medicine, Chang Gung University, Taoyuan City, Taiwan R.O.C.

3 Department of Computer Science and Information Engineering, Chang Gung University, Gueishan District, No. 259, Wen-Hwa 1st Road, Taoyuan City, Taiwan R.O.C. 
Recently, noninvasive oropharyngeal swallowing study tools are being used, and various noninvasive sensors are used to detect and record thyroid cartilage excursion from the skin surface. These sensors include piezoelectric sensors, accelerators, bend sensors, force-sensing resistor (FSR) sensors [1, 4-9], and sonography [10]. Concurrently, a stethoscope with an amplifier or microphone can record and collect the sounds of swallowing [11-13]. Each sensor and method has strengths, but different laboratories have objectives, conditions, and other factors (e.g., being familiar with software or hardware already in their laboratory), which may help them to make a choice between different sensors.

Our team used piezoelectric [7, 9] and FSR [1, 2, 8, 14] sensors to monitor thyroid cartilage during swallowing. However, finding new sensors and comparing their results with those from videofluoroscopic swallowing studies (VFSS) is valuable with regard to research and clinical applications.

Detecting thyroid cartilage excursion is a key feature of noninvasive swallowing study. A study demonstrated that excursion of thyroid cartilage is synchronized with hyoid motion during VFSS [15]. When swallowing responses start, the hyoid bone moves upward and forward promptly, remains at the highest point, and then, returns to its initial position [15-17]. Hyoid bone and thyroid cartilage demonstrate synchronized movement during swallowing, referred to as hyolaryngeal movement $[18,19]$ or hyolaryngeal excursion [20]. Notably, these sequential movements are related to swallowing respiratory pause and to the timing and duration of upper esophageal sphincter relaxation during swallowing. These timely coordinated movements allow the bolus to pass the cricopharyngeal sphincter smoothly and securely $[6,21]$. Therefore, monitoring and analyzing hyoid motion during VFSS is crucial. Monitoring thyroid cartilage excursion in noninvasive swallowing studies using surface sensors is also vital.

The results from noninvasive swallowing studies using FSR sensors to detect the thyroid cartilage excursion, however, were not correlated with those from VFSS. Therefore, our team synchronized these two systems to verify if the temporal parameters between the systems were correlated and postulated that laryngeal excursion detected using FSR sensors is synchronized with the hyoid motion during VFSS. This study aimed to identify the relationship between the temporal swallowing parameters of laryngeal excursion signals detected using FSR sensors and hyoid motion images detected during VFSS, which were recorded simultaneously in young volunteers without dysphagia.

\section{Materials and Methods}

\section{Volunteers and Ethical Approval}

The Ethics Committee of Chang-Gung Memorial Hospital approved the study protocol (No. 201602016B0C101). Each participant was introduced to and thoroughly explained the study's aims and procedures and asked to sign the informed consent form.

All participants were young and healthy. Exclusion criteria included a history of dysphagia, cardiopulmonary disease, neurological disease, chronic indigestion disorder, diseases of the head and neck, and smoking in the previous 5 years. All volunteers were administered the Functional Oral Intake Scale (FOIS) [22] and Eating Assessment Tool (EAT-10) [23]; the results on neither of the scales demonstrated dysphagia. In total, 29 healthy volunteers [15 men (mean age: $28.0 \pm 4.1$ years) and 14 women (mean age: $28.4 \pm 4.2$ years)] were recruited. The participant characteristics are listed in Table 1.

\section{Instruments (Hardware and Software)}

The ambulatory noninvasive swallowing respiration assessment system based on the MP150 system (MP150 system, Biopac, Goleta, CA, USA) was sent to radiological suites and set up for simultaneous recording by using a VFSS equipment (Luminos; Siemens).

\section{Biopac System}

Two FSR sensors were used-one to detect thumb pressing and the other to detect thyroid cartilage excursion. Noninvasive swallowing and respiration coordination signal monitoring based on the Biopac system was set up in our laboratory when FSR sensors were used to detect thyroid cartilage excursion in previous studies [1, 3, 7-9]. To collect the test data for the correlation of temporal parameters of signals detected using FSR sensors with images recorded in VFSS, a noninvasive swallowing respiration system based on Biopac, including recording of submental sEMG and nasal airflow

Table 1 Characteristics of young healthy volunteers without dysphagia

\begin{tabular}{llll}
\hline & M (No:15) & F (No:14) & $P$-vale \\
\hline Age (year/old) & $28.00 \pm 4.13$ & $28.43 \pm 4.22$ & 0.784 \\
BMI $\left(\mathrm{kg} / \mathrm{m}^{2}\right)$ & $22.69 \pm 4.90$ & $22.48 \pm 1.81$ & 0.124 \\
Height $(\mathrm{cm})$ & $173.8 \pm 6.0$ & $163.4 \pm 5.1$ & 0.000 \\
Weight $(\mathrm{kgs})$ & $68.60 \pm 15.85$ & $54.86 \pm 7.37$ & 0.006 \\
\hline
\end{tabular}

$M$ male, $F$ female, y/o year old, $B M I$ body mass index 
for respiration, were also recorded for verification. Moreover, one FSR sensor was fixed at the thyroid cartilage level on the midline of the anterior neck skin surface to detect thyroid cartilage excursion [1]. Another FSR sensor was fixed on the upper side of a handheld stick which was designed as an event marker to detect thumb pressing motions (Fig. 1). The swallowing signals using noninvasive systems and images using VFSS were also recorded simultaneously, and the motion of thumb pressing signals were used as time reference points for the two swallowing study systems.

\section{Vfss}

For the VFSS, the recording speed was 30 frames/s in the lateral view and the time duration was $1 / 30$-s interval per image.

\section{Simultaneously Recording Of Swallowing-Related Thyroid Cartilage Excursion Signals Using MP150 System And Hyoid Motion During VFSS}

To overcome the problem of synchronizing the two systems Biopac system and VFSS, another FSR sensor, on the upper end of a handheld stick designed to detect a thumb pressing, was used. These thumb pressing reference points enabled the analysis of timepoints and durations between the two systems. The noninvasive oropharyngeal swallowing signals, including those detected using FSR sensors, were

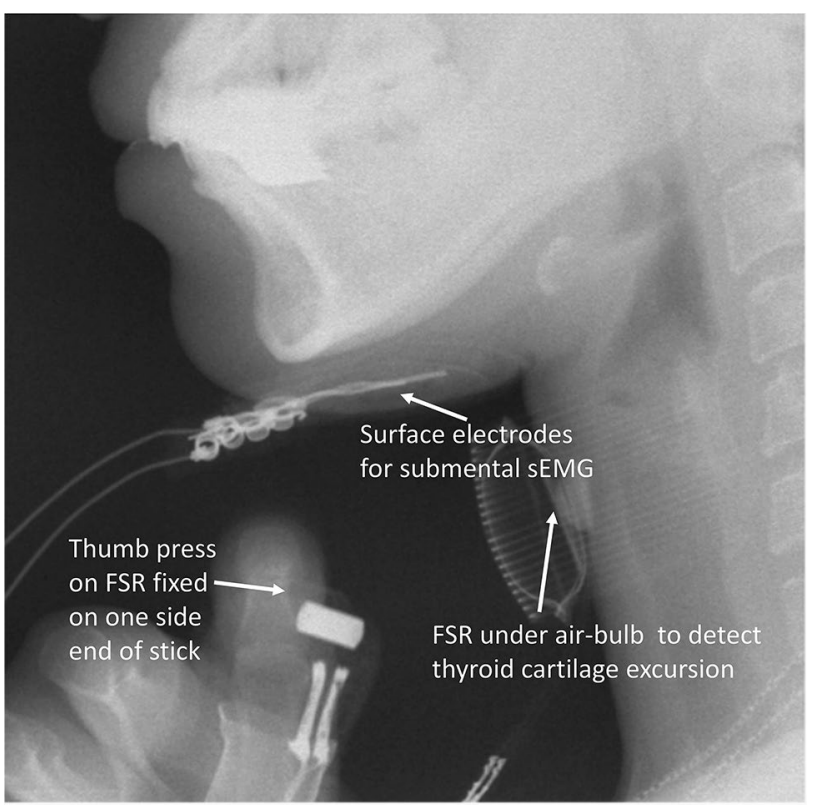

Fig. 1 FSR sensor attached to the side of a small handheld stick to detect thumb pressing, and FSR sensor under an air bulb to detect thyroid excursion under VFSS simultaneously recorded during VFSS and analyzed offline later using LabView (version 2010; Mathworks, Natick, MA, USA).

\section{Software}

Acqknowledge (version 5.0; Biopac) for the MP150 system was used for recording swallowing signals, and LabView was used for the subsequent offline analysis.

\section{Study Procedures}

To validate FSR sensor detection of temporal swallowing parameters, an ambulatory noninvasive swallowing study system (based on the MP150 system) was used and a VFSS was performed simultaneously in radiological suites. In this study, a specially designed FSR sensor on one side of a small stick was held in the palm to detect thumb pressings as the reference time events for analysis. Volunteers were equipped with two ECG adhesive electrodes for submental sEMG, a nasal cannula for respiration, an FSR sensor for the laryngeal excursion, and an FSR sensor for thumb pressing-all of which were connected onto the MP150 system. These validation study procedures included an FSR sensor held in the volunteer's hand, which was pressed with the thumb twice-before swallowing and once after swallowingand another FSR sensor was fixed onto the anterior neck at thyroid cartilage level to detect thyroid cartilage excursion during 5-mL barium swallowing in VFSS. During VFSS, the volunteers sat on a chair with their head vertical to the Frankfort plane. Few small water boluses at room temperature were dispensed to volunteers for the setting up a noninvasive measurement system and to familiarize the volunteers with the test procedure. Liquid barium (30\% wt/volume \%; $5 \mathrm{~mL}$ ) bolus was administered using a $20-\mathrm{mL}$ disposable syringe into the mouth of the volunteers. All participants were instructed to hold the FSR sensor fixed on one side of small stick in their hands and hold the fed 5-mL barium bolus in their mouths; they were then asked to press the FSR sensor and swallow the barium bolus on verbal instruction by the radiologist in the following order: "press" (thumb press the FSR sensor on the stick), "press," "swallow," and "press". In total, four separate single boluses of 5-mL barium were recorded each individually by using the two systems simultaneously. In the lateral view in the VFSS, the dynamic images of oropharyngeal swallowing were recorded with 30 images/s. All VFSS videos were recorded and saved in an picture archiving and communication system (PACS). After the study was completed, the copies of VFSS videos from PACS were exported for later offline analysis. The recorded swallowing and respiration signals were exported for subsequent offline analysis (Fig. 2). 


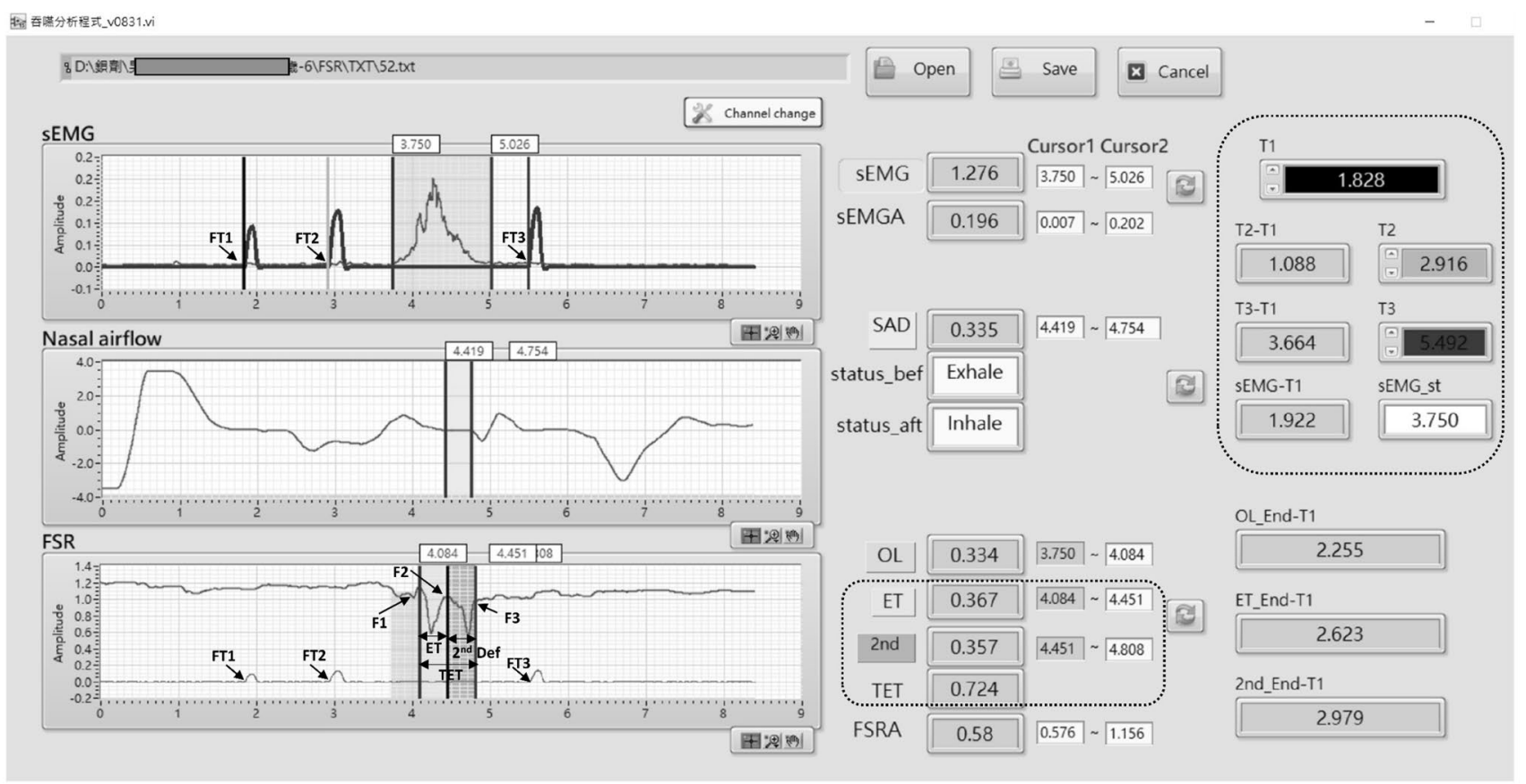

Fig. 2 LabView for noninvasive swallowing and respiration parameter analysis and the three events of thumb pressing on FSR sensor

The total radiation exposure time was controlled to $<2$ min [4] because the dose of radiation in similar circumstances is approximately one-third of clinical VFSS in patients with dysphagia at our hospital, which is approved by our radiation safety committee and institutional review board. No choking events were noted during the study. No volunteer displayed signs of dysphagia or aspiration during the VFSS combined with noninvasive swallowing study. All the participants exhibited normal tongue base retraction, epiglottis inversion, epiglottis seal, and upper esophageal sphincter (UES) opening during barium bolus VFSS.

\section{Defining Time Events (Onset Timepoints) and Durations on VFSS Images and Noninvasive Swallowing Assessment Tool Signals During Swallowing}

A single-frame analysis was used in our VFSS image analysis [24]. To calculate the time and duration of hyoid motion [25], the frame number of each image recording was used to calculate the time when the action occurred as well as the onset timepoint and duration of each motion.

To accurately analyze hyoid displacement, the investigators underwent 3 months of extensive training on the biomechanical analysis of swallowing and VFSS before joining this investigation. Two independent trained investigators manually selected three target frames from the timeframe set for the FSR sensor and three target frames of hyoid bone-related sequence movement during swallowing including the frame of movement initiation, movement of the hyoid bone toward the frame at uppermost point, and movement back to the resting position. The resting and uppermost frames of the hyoid bone movement during swallowing were selected only after multiple observations of the entire swallow in real-time and after frame-by-frame inspection. The resting position of the hyoid bone was marked as the position that hyoid was in the moment before the bolus was propelled into the pharynx from the oral cavity. After each frame was defined, the time duration between the three targeted swallowing frames and three thumb pressing frames were calculated (Figs. 2 and 3). There were some disagreements in the frame-by-frame reading results between two investigators, which were resolved after the results were reanalyzed and discussed to reach a consensus.

\section{Definitions of Hyoid Bone Movement Events Observed During VFSS}

We defined three events of hyoid bone movement (V1, V2, and V3) during VFSS [4] along with three thumb press signals on the handheld FSR sensor (VT1, VT2, and VT3). Accordingly, the onset times of six events (Table 2) and five periods (Table 3) were defined and analyzed. 


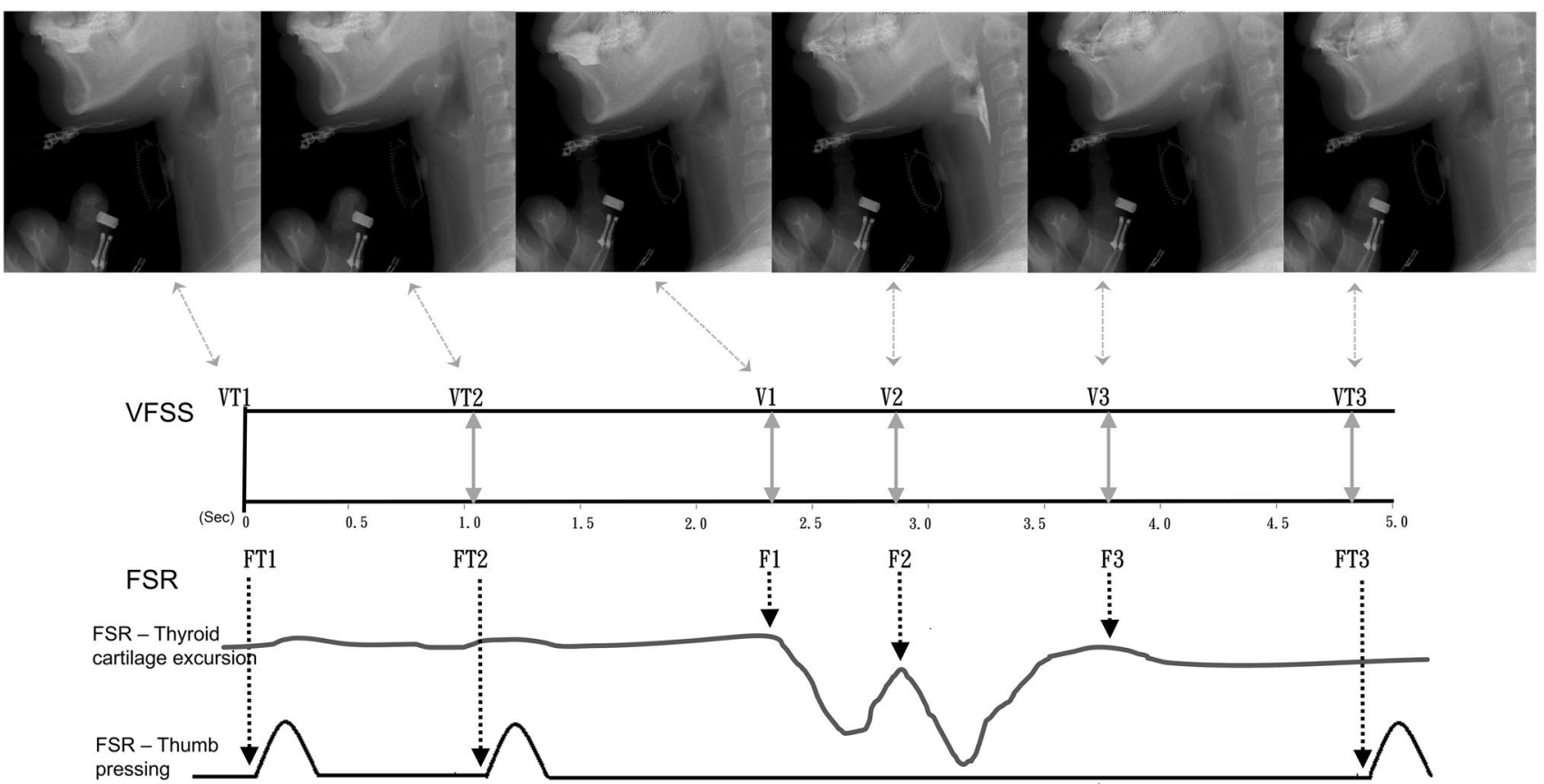

Fig. 3 Timepoints on the signal waveform from the FSR sensor (FT1-FT3, F1-F3) and VF events (VT1-VT3, V1-V3)

Table 2 Definition of six VFSS events

\begin{tabular}{ll}
\hline VF 6 events & Physiological activity of the hyoid bone \\
1. VT1 & First thumb press on handheld FSR sensor \\
2. VT2 & Second thumb press on handheld FSR sensor \\
3. V1 & Onset of movement of the hyoid bone (begin to move from initial position) \\
4. V2 & Onset of stationary phase of the hyoid bone (upmost position) \\
5. V3 & End of offset of movement of the hyoid bone (back to initial position) \\
6. VT3 & Third thumb press on handheld FSR sensor \\
\hline
\end{tabular}

Table 3 Definition of 5 time periods (duration)

\footnotetext{
\#1 VT1-VT2 First thumb press handheld FSR sensor to second thumb press handheld FSR sensor \#2 VT1-V1 First thumb press handheld FSR sensor to the onset of movement of the hyoid bone

\#3 VT1-V2 First thumb press handheld FSR sensor to the onset of stationary phase of the hyoid bone

\#4 VT1-V3 First thumb press handheld FSR sensor to the offset of stationary phase of the hyoid bone

\#5 VT1-VT3 First thumb press handheld FSR sensor to third thumb press handheld FSR sensor
}

\section{Defining the waveforms of thyroid cartilage excursion that detected and recorded using FSR sensor and the waveforms of thumb pressed on the handheld FSR sensor (Figs. 2 and 3)}

FSR sensors were used to detect thyroid cartilage excursion during swallowing. A W-shaped waveform with two "V"s was recorded. F1 is the onset time of $\mathrm{W}, \mathrm{F} 2$ is the turning point of $\mathrm{W}$, and $\mathrm{F} 3$ is the offset time. A semiautomatic analysis software based on LabView was used, which detected and analyzed all three swallowing-related signals (nasal airflow, submental sEMG, and thyroid cartilage excursion) locked in the same frame of three different traces [26] and combined with the three signal trace from thumb pressing on FSR sensor. The three individual onset times of thumb pressing on the handheld FSR sensor were represented as T1, T2, and $\mathrm{T} 3$, comprising two pressings (T1 and T2) before swallowing action and one (T3) after swallowing (Figs. 2 and 3). The onsets of T1, T2, and T3 are defined and marked in Fig. 2; these were overlapped in the uppermost trace panel of submental sEMG. After the signals were defined and analyzed using LabView (Fig. 2), the data were exported to an MS 
Excel file for subsequent statistical analysis. This automatic output to an MS Excel file saved time and circumvented human error in the process of data entry.

\section{Data Analysis}

The point VT1 in VFSS and FT1 by FSR sensor were defined as timepoint zero (Fig. 3). Consecutively, the timepoints of VT2, VT3, V1, V2, and V3 corresponded to the FT2, FT3, F1, F2, and F3, respectively. These were all critical onset times for specially defined events in both swallowing study images.

\section{Statistical Analysis}

Statistical analyses were performed using SPSS statistical package (version 12.0; SPSS, Inc., Chicago, IL, USS). To determine the correlation between the hyoid motion during VFSS and thyroid cartilage excursion by using FSR in a noninvasive system, Pearson's correlation coefficients ( $(\mathrm{s})$ were calculated. Statistical significance between the parameters of the two study systems was taken as a $p$-value of $<0.01$ for a two-tailed T test.

\section{Results}

The temporal parameters hyoid motion detected during VFSS and thyroid cartilage excursion detected using FSR sensors demonstrated highly significant correlation $(R=0.838-0.990$, all $p<0.001$; data not shown). No significant differences were noted in the temporal parameters between the two tests for each corresponding time duration (VT1-T2 vs FT1-F2, VT1-T3 vs FT1-T3, VT1-V2 vs FT1-F2, and VT1-V3 vs FT1-F3), except for the onset of hyoid motion (VT1-V1) and of thyroid cartilage excursion (FT1-F1), which demonstrated a significant difference $(p=0.004$; data not shown).

We next analyzed the data in separate groups of men and women. The defined temporal parameters for analysis between VFSS and noninvasive systems demonstrated strong correlations in men $(R=0.953-0.999$; all $p<0.001)$. In women, the correlation was strong for all $(R=0.813-0.982$; all $p<0.001$ ), except VT1-V1 and FT1-F1, which displayed moderate correlation $(R=0.648 ; p=0.001$; Table 4$)$. In men, the five corresponding time durations between the two tests was statistically nonsignificant, whereas only VT1-V1 and FT1-F1 displayed significant differences in women $(p=0.001$; Table 5).

However, a limitation of this study is that the time scales of the two swallowing measurement systems were different. The time interval by the noninvasive system was $0.001 \mathrm{~s}$, but the interval for VFSS was $0.033 \mathrm{~s}$. This scale difference
Table 4 Pearson correlation of temporal parameters between VFSS and noninvasive swallowing assessment using FSR sensor in young volunteers without dysphagia [ $n=29$ (Men: 15, Women: 14)]

\begin{tabular}{llllll}
\hline & \multicolumn{2}{l}{ Male (No:15) } & & \multicolumn{2}{l}{ Female (No:14) } \\
\cline { 2 - 3 } & $\mathrm{R}$ & $P$ value & & $\mathrm{R}$ & $P$-value \\
\hline VT1-T2 vs FT1-T2 & 0.998 & $<0.001^{*}$ & & 0.982 & $<0.001^{*}$ \\
VT1-T3 vs FT1-T3 & 0.999 & $<0.001^{*}$ & & 0.826 & $<0.001^{*}$ \\
VT1-V1 vs FT1-F1 & 0.960 & $<0.001^{*}$ & & 0.648 & $<0.001^{*}$ \\
VT1-V2 vs FT1-F2 & 0.984 & $<0.001^{*}$ & & 0.955 & $<0.001^{*}$ \\
VT1-V3 vs FT1-F3 & 0.953 & $<0.001^{*}$ & & 0.813 & $<0.001^{*}$ \\
\hline
\end{tabular}

$* P$-value $<0.01$

may have affected the correlation analysis results between the two measurement systems.

\section{Discussion}

This study was the first to analyze concurrent noninvasive sensors and VFSS recordings of swallowing signals. A previous study investigating swallowing signals using a bend sensor to detect thyroid cartilage motion and VFSS to monitor hyoid motion and demonstrated a positive correlation in their results; however, in this study, swallowing signals detection using bend sensors and VFSS was not simultaneously, and it recruited only six young healthy men without dysphagia were recruited, but no women [4]. Another study synchronized the piezoelectric pulse transducer (PPT) waveforms to VFSS recordings of pharyngeal swallowing events in three healthy men [27]. Therefore, thus far, data on the correlation between surface sensors waveforms of swallowing and VFSS as well as a lack of synchronized and simultaneous recordings of two swallowing study methods have been limited.

In the current study, we recruited more normal healthy young volunteers - both men and women - and determined that both men and women displayed strong correlation of temporal parameters between the two swallowing test systems (the thyroid cartilage excursion using FSR through the noninvasive method and the hyoid motion during VFSS). There were no significant differences in temporal parameters between the two systems, except for that in one temporal parameter (VT1-V1 to FT1-F1) in women that displayed a statistically significant difference but a high correlation. Unlike in the study on the correlation of bend sensor and VFSS [4], we simultaneously recorded swallowing events with two systems.

Findings on the effects of gender on swallowing thus far have been inconclusive: some have indicated differences in swallowing parameters between genders, whereas have not [28-33]. These inconclusive findings complicate the data 
Table 5 Test comparison of temporal parameters of swallowing signals between VFSS and FSR sensor in young volunteers without dysphagia $(n=29)$

\begin{tabular}{|c|c|c|c|c|c|}
\hline \multicolumn{3}{|l|}{ Male (No:15) } & \multicolumn{3}{|l|}{ Female (No:14) } \\
\hline VFSS & FSR & $P$-value & VFSS & FSR & $P$-value \\
\hline $\begin{array}{l}\text { VT1-T2 } \\
1.153 \pm 0.394(\mathrm{~S})\end{array}$ & $\begin{array}{l}\text { FT1-T2 } \\
1.153 \pm 0.392(\mathrm{~S})\end{array}$ & 0.995 & $\begin{array}{l}\text { VT1-T2 } \\
0.996 \pm 0.439(\mathrm{~S})\end{array}$ & $\begin{array}{l}\text { FT1-T2 } \\
1.008 \pm 0.428(S)\end{array}$ & 0.884 \\
\hline $\begin{array}{l}\text { VT1-T3 } \\
4.494 \pm 1.075(\mathrm{~S})\end{array}$ & $\begin{array}{l}\text { FT1-T3 } \\
4.494 \pm 1.073(\mathrm{~S})\end{array}$ & 0.997 & $\begin{array}{l}\text { VT1-T3 } \\
4.678 \pm 0.830(\mathrm{~S})\end{array}$ & $\begin{array}{l}\text { FT1-T3 } \\
4.783 \pm 0.875(\mathrm{~S})\end{array}$ & 0.521 \\
\hline $\begin{array}{l}\text { VT1-V1 } \\
2.375 \pm 0.791(\mathrm{~S})\end{array}$ & $\begin{array}{l}\text { FT1-F1 } \\
2.553 \pm 0.789(\mathrm{~S})\end{array}$ & 0.267 & $\begin{array}{l}\text { VT1-V1 } \\
2.241 \pm 0.580(\mathrm{~S})\end{array}$ & $\begin{array}{l}\mathrm{FT} 1-\mathrm{F} 1 \\
2.630 \pm 0.575(\mathrm{~S})\end{array}$ & $0.001 *$ \\
\hline $\begin{array}{l}\text { VT1-V2 } \\
2.834 \pm 0.782(\mathrm{~S})\end{array}$ & $\begin{array}{l}\text { FT1-F2 } \\
2.971 \pm 0.731(\mathrm{~S})\end{array}$ & 0.451 & $\begin{array}{l}\text { VT1-V2 } \\
2.916 \pm 0.429(\mathrm{~S})\end{array}$ & $\begin{array}{l}\text { FT1-F2 } \\
3.128 \pm 0.458(\mathrm{~S})\end{array}$ & 0.032 \\
\hline $\begin{array}{l}\text { FT1-V3 } \\
3.697 \pm 0.820(\mathrm{~S})\end{array}$ & $\begin{array}{l}\text { FT1-F3 } \\
3.808 \pm 0.809(\mathrm{~S})\end{array}$ & 0.501 & $\begin{array}{l}\text { FT1-V3 } \\
3.717 \pm 0.595(\mathrm{~S})\end{array}$ & $\begin{array}{l}\text { FT1-F3 } \\
3.910 \pm 0.584(\mathrm{~S})\end{array}$ & 0.099 \\
\hline
\end{tabular}

$S$ second

$* p<0.01$ analysis and the interpretation of our results, necessitating more data for confirmation. Our previous study recruited 19 young normal volunteers ( 9 men and 10 women) to compare the difference of two placement levels of piezoelectric sensors for detecting thyroid cartilage excursion as well as understanding the sex differences. Piezoelectric sensor recordings and surface electromyography were collected during the swallowing process. The sensor placement at the thyroid cartilage level afforded a greater amplitude of laryngeal excursion than did that at the cricothyroidotomy level. Furthermore, in women, oropharyngeal phase-related parameters differed significantly more at the thyroid cartilage level than at the cricothyroidotomy level [9]. In this study, the FSR sensor to monitor the thyroid cartilage excursion was placed at the thyroid cartilage level, which may account in part for our finding that women did not display a strong correlation between the FSR and VFSS results.

When simultaneously recording the swallowing signals of a noninvasive FSR sensor swallowing study system and VFSS, some technical issues need to be solved using hardware and software. To synchronize the two systems, a handheld stick with an FSR sensor, connected to one of the multiple MP150 system channels, was used in this study. Our handheld stick design could be used in future swallowing studies to create timepoint references to aid in synchronizing FSR with VFSS or other recording methods.

This noninvasive swallowing study method using FSR sensors to detect thyroid cartilage excursion of swallowing is reliable and efficient at indirectly detecting the temporal parameters of oropharyngeal swallowing, which has uses in clinical application and future studies. This system has multiple channels to simultaneously record, and time-lock, swallowing-related physiological signals including submental sEMG, nasal airflow, and uncalibrated measurement of global laryngeal excursion, which is adequate to detect a swallowing event. Swallowing events were analyzed but the detailed parameters were not. The reliability of the detection of swallowing events has been demonstrated, and thus, this noninvasive system can be used to analyze piecemeal deglutition and dysphagia limits [26]. These findings are similar to those of a previous report that PPT waveforms corresponded to pharyngeal phase swallowing under VFSS [27]. Moreover, with nasal airflow monitoring, our noninvasive system can be used in studies of swallowing and respiration coordination which include swallowing respiratory pause and pre- and post-swallowing respiratory phase patterns. Furthermore, a stepwise volume increase in swallowing water bolus protocol would not only be beneficial for safety but also could be used to detect the piecemeal deglutition that appears after a water bolus of $<20 \mathrm{~mL}$ administered [1-3, $7,14]$. Another principal outcome is that the protocol and analysis methods in this noninvasive swallowing and respiration study system can be used to detect subtle, subclinical [1], and early [2] mild dysphagia. Furthermore, this system can be used for the older people [7], during follow-up [3], and in treatment effectiveness studies [14]. Regarding improvements and applications, advancements in hardware and software are crucial. We have made our system ambulatory and plan to create a portable version. For the pediatric population, we plan to find small surface sensors to detect thyroid cartilage excursion during swallowing.

Swallowing study objective instruments all have various advantages and limitations. The numerous facets of swallowing function studies include temporal parameters [27, 29], dynamic biomechanics [19, 34], and pressure measurement [35]. One study tool cannot be substituted for another, and using multiple study tools can assist, supplement, or compensate for the other swallowing study tools $[35,36]$. Therefore, using different swallowing study instruments to examine various facets of swallowing could prove valuable with regards to clinical applications and research. Noninvasive swallowing detection systems is an objective tool that is gaining attention because it demonstrates promising results and thus should be investigated and developed further. The 
improvement and diversification of the noninvasive swallowing detection system is crucial.

The noninvasive swallowing study without radiation exposure is suitable for posttherapy monitoring of the immediate effects. Furthermore, the advantages of noninvasive swallowing study systems include the possibility of a larger quantity of swallowing events data that can be collected for a cross-section study, a larger number of repeated measurements over a short period in follow-up studies, and studies for swallowing data collection over a long follow-up duration. It is also useful in long-term objective follow-up studies. Another valuable potential application of a noninvasive swallowing system is in feedback training $[37,38]$. Our system had already been programmed to monitor nasal airflow for respiration coordination during swallowing, which can be used in visual feedback training with signals displayed on screen.

Noninvasive systems are, however, not without limitations. The noninvasive system cannot record through the oropharyngeal anatomic structures and hyoid displacement, UES relaxation, bolus passage, penetration, aspiration, and residuals after swallowing were recorded during VFSS [39]. These findings have clinical applicability in making decisions for swallowing training programs and deciding whether to remove nasogastric tubes. Noninvasive swallowing study cannot replace VFSS. The noninvasive swallowing study was developed to complete the information obtained from analysis with VFSS [10, 35, 36, 40-47].

Developing automatic analysis software systems to analyze the dynamic images and temporal parameters of swallowing would be valuable in both VFSS and noninvasive swallowing study systems. Using artificial intelligence to solve this semiautomatic analysis problem may lead noninvasive swallowing studies to another frontier.

\section{Conclusions}

This is the first study to concurrently record noninvasive swallowing signals and perform VFSS. We provided a new method for researchers to conduct correlation studies between noninvasive swallowing studies and VFSS.

Our results demonstrated a strong correlation in the temporal parameters of swallowing between noninvasive surface sensors and VFSS. This is crucial for further usage of this noninvasive swallowing study tool in studies and clinically.

Funding The study was financially supported by Grants from the National Science Council, Taiwan (MOST 107-2314-B-182 -010; MOST 108-2221-E-182-016-MY3) and Chang Gung Memorial Hospital, Taiwan (CMRPD2H0141-3).

\section{Compliance with ethical standards}

Conflict of interest The authors have no other funding, financial relationships, or conflicts of interest to disclose.

Open Access This article is licensed under a Creative Commons Attribution 4.0 International License, which permits use, sharing, adaptation, distribution and reproduction in any medium or format, as long as you give appropriate credit to the original author(s) and the source, provide a link to the Creative Commons licence, and indicate if changes were made. The images or other third party material in this article are included in the article's Creative Commons licence, unless indicated otherwise in a credit line to the material. If material is not included in the article's Creative Commons licence and your intended use is not permitted by statutory regulation or exceeds the permitted use, you will need to obtain permission directly from the copyright holder. To view a copy of this licence, visit http://creativecommons.org/licenses/by/4.0/.

\section{References}

1. Wang CM, Li HY, Lee LA, et al. Non-invasive assessment of swallowing and respiration coordination for the OSA patient. Dysphagia. 2016;31:771-80.

2. Wang CM, Shieh WY, Weng YH, et al. Non-invasive assessment determine the swallowing and respiration dysfunction in early Parkinson's disease. Parkinsonism Relat Disord. 2017;42:22-7.

3. Wang CM, Shieh WY, Chen JY, Wu YR. Integrated non-invasive measurements reveal swallowing and respiration coordination recovery after unilateral stroke. Neurogastroenterol Motil. 2015;27:1398-408

4. Li Q, Hori K, Minagi Y, et al. Development of a system to monitor laryngeal movement during swallowing using a bend sensor. PLoS ONE. 2013;8:e70850.

5. Ono T, Hori K, Masuda Y, Hayashi T. Recent advances in sensing oropharyngeal swallowing function in Japan. Sensors. 2010;10:176-202.

6. Ertekin C, Pehlivan M, Aydogdu I, et al. An electrophysiological investigation of deglutition in man. Muscle Nerve. 1995;18:1177-86.

7. Wang CM, Chen JY, Chuang CC, et al. Aging-related changes in swallowing, and in the coordination of swallowing and respiration determined by novel non-invasive measurement techniques. Geriatr Gerontol Int. 2015;15:736-44

8. Shieh WY, Wang CM, Chang CS. Development of a portable non-invasive swallowing and respiration assessment device. Sensors. 2015;15:12428-53.

9. Wang CM, Chuang CC, Chen PC, et al. Noninvasive swallowing test for young healthy adults: finding the best location to monitor thyroid cartilage movements. J Med Biol Engin. 2014:34:393-8.

10. Hsiao MY, Chang YC, Chen WS, et al. Application of ultrasonography in assessing oropharyngeal dysphagia in stroke patients. Ultrasound Med Biol. 2012;38:1522-8.

11. Moriniere S, Beutter P, Boiron M. Sound component duration of healthy human pharyngoesophageal swallowing: a gender comparison study. Dysphagia. 2006;21:175-82.

12. Youmans SR, Stierwalt JA. An acoustic profile of normal swallowing. Dysphagia. 2005;20:195-209.

13. Moriniere S, Boiron M, Alison D, et al. Origin of the sound components during pharyngeal swallowing in normal subjects. Dysphagia. 2008;23:267-73. 
14. Wang CM, Shieh WY, Ho CS, et al. Home-based orolingual exercise improves the coordination of swallowing and respiration in early parkinson disease: a quasi-experimental before-and-after exercise program study. Front Neurol. 2018;9:624.

15. Yokoyama M, Mitomi N, Tetsuka K, et al. Role of laryngeal movement and effect of aging on swallowing pressure in the pharynx and upper esophageal sphincter. Laryngoscope. 2000;110:434-9.

16. Palmer JB, Tanaka E, Ensrud E. Motions of the posterior pharyngeal wall in human swallowing: a quantitative videofluorographic study. Arch Phys Med Rehabil. 2000;81:1520-6.

17. Ashida I, Miyaoka S, Miyaoka Y. Comparison of video-recorded laryngeal movements during swallowing by normal young men with piezoelectric sensor and electromyographic signals. J Med Eng Technol. 2009;33:496-501.

18. Humbert IA, Christopherson H, Lokhande A, et al. Human hyolaryngeal movements show adaptive motor learning during swallowing. Dysphagia. 2013;28:139-45.

19. Kim SJ, Han TR, Kwon TK. Kinematic analysis of hyolaryngeal complex movement in patients with dysphagia development after pneumonectomy. Thorac Cardiovasc Surg. 2010;58:108-12.

20. Zoratto DC, Chau T, Steele CM. Hyolaryngeal excursion as the physiological source of swallowing accelerometry signals. Physiol Meas. 2010;31:843-55.

21. Ertekin C, Celik M, Secil Y, et al. The electromyographic behavior of the thyroarytenoid muscle during swallowing. J Clin Gastroenterol. 2000;30:274-80.

22. Crary MA, Mann GD, Groher ME. Initial psychometric assessment of a functional oral intake scale for dysphagia in stroke patients. Arch Phys Med Rehabil. 2005;86:1516-20.

23. Belafsky PC, Mouadeb DA, Rees CJ, et al. Validity and reliability of the eating assessment tool (EAT-10). Ann Otol. Rhinol Laryngol. 2008;117:919-24.

24. Taniguchi H, Tsukada T, Ootaki S, et al. Correspondence between food consistency and suprahyoid muscle activity, tongue pressure, and bolus transit times during the oropharyngeal phase of swallowing. J Appl Physiol. 2008;105:791-9.

25. Hori K, Taniguchi $\mathrm{H}$, Hayashi $\mathrm{H}$, et al. Role of tongue pressure production in oropharyngeal swallow biomechanics. Physiol Rep. 2013;1:e00167.

26. Wang CM, Tsai TT, Wang SH, Wu YR. Does the M.D. Anderson dysphagia inventory correlate with dysphagia-limit and the unified parkinson disease rating scale in early-stage parkinson's disease? J Formos Med Assoc. 2019;119(1):247-53.

27. Toyosato A, Nomura S, Igarashi A, et al. A relation between the piezoelectric pulse transducer waveforms and food bolus passage during pharyngeal phase of swallow. Prosthodontic Res Pract. 2007;6:272-5.

28. Cichero JA, Murdoch BE. Acoustic signature of the normal swallow: characterization by age, gender, and bolus volume. Ann Otol Rhinol Laryngol. 2002;111:623-32.

29. Butler SG, Stuart A, Castell D, et al. Effects of age, gender, bolus condition, viscosity, and volume on pharyngeal and upper esophageal sphincter pressure and temporal measurements during swallowing. J Speech Lang Hear Res. 2009;52:240-53.

30. Logemann JA, Pauloski BR, Rademaker AW, et al. Temporal and biomechanical characteristics of oropharyngeal swallow in younger and older men. J Speech Lang Hear Res. 2000;43:1264-74.

31. Kim Y, McCullough GH, Asp CW. Temporal measurements of pharyngeal swallowing in normal populations. Dysphagia. 2005;20:290-6.

32. Lof GL, Robbins J. Test-retest variability in normal swallowing. Dysphagia. 1990;4:236-42.

33. Robbins J, Hamilton JW, Lof GL, Kempster GB. Oropharyngeal swallowing in normal adults of different ages. Gastroenterology. 1992;103:823-9.
34. van der Kruis JG, Baijens LW, Speyer R, Zwijnenberg I. Biomechanical analysis of hyoid bone displacement in videofluoroscopy: a systematic review of intervention effects. Dysphagia. 2011;26:171-82.

35. Huckabee ML, Macrae P, Lamvik K. expanding instrumental options for dysphagia diagnosis and research: ultrasound and manometry. Folia Phoniatr Logop. 2015;67:269-84.

36. Reginelli A, Iacobellis F, Del Vecchio L, et al. VFMSS findings in elderly dysphagic patients: our experience. BMC Surg. 2013;13(Suppl 2):S54.

37. Tran TTA, Harris BM, Pearson WG Jr. Improvements resulting from respiratory-swallow phase training visualized in patient-specific computational analysis of swallowing mechanics. Comput Methods Biomech Biomed Eng Imaging Vis. 2018;6:532-8.

38. Martin-Harris B, Garand KLF, McFarland D. Optimizing respiratory-swallowing coordination in patients with oropharyngeal head and neck cancer. Perspect ASHA Spec Interest Groups. 2017;2:103-10

39. Steele CM, Grace-Martin K. Reflections on clinical and statistical use of the penetration-aspiration scale. Dysphagia. 2017;32:601-16.

40. Sejdic E, Dudik JM, Kurosu A, et al. Understanding differences between healthy swallows and penetration-aspiration swallows via compressive sensing of tri-axial swallowing accelerometry signals. Proc SPIE Int Soc Opt Eng. 2014;9190:91090M.

41. Steele CM, Sejdic E, Chau T. Noninvasive detection of thin-liquid aspiration using dual-axis swallowing accelerometry. Dysphagia. 2013;28:105-12.

42. Jayatilake D, Ueno T, Teramoto Y, et al. Smartphone-based realtime assessment of swallowing ability from the swallowing sound. IEEE J Transl Eng Health Med. 2015;3:2900310.

43. Afkari S. Measuring frequency of spontaneous swallowing. Australas Phys Eng Sci Med. 2007;30:313-7.

44. Lee J, Blain S, Casas M, et al. A radial basis function classifier for pediatric aspiration detection. Conf Proc IEEE Eng Med Biol Soc. 2006;1:3553-6.

45. Borr C, Hielscher-Fastabend M, Lucking A. Reliability and validity of cervical auscultation. Dysphagia. 2007;22:225-34.

46. Santamato A, Panza F, Solfrizzi V, et al. Acoustic analysis of swallowing sounds: a new technique for assessing dysphagia. $\mathrm{J}$ Rehabil Med. 2009;41:639-45.

47. O'Horo JC, Rogus-Pulia N, Garcia-Arguello L, et al. Bedside diagnosis of dysphagia: a systematic review. J Hosp Med. $2015 ; 10: 256-65$

Publisher's Note Springer Nature remains neutral with regard to jurisdictional claims in published maps and institutional affiliations.

\section{Chin-Man Wang MD}

Chao-Jan Wang MD

Wann-Yun Shieh $\mathrm{PhD}$

Yen-Chia Chen MD/MPH

Wei-Jen Cheng MD

Wei-Han Chang MD 